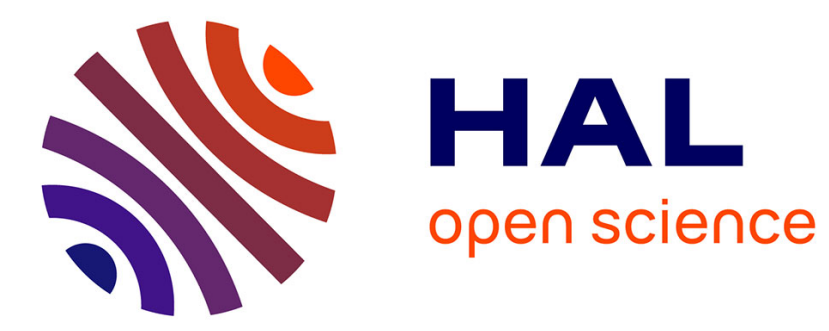

\title{
Violences conjugales, famille, vulnérabilité
}

Laurie Laufer, Thamy Ayouch

\section{To cite this version:}

Laurie Laufer, Thamy Ayouch. Violences conjugales, famille, vulnérabilité. Topique - Revue freudienne, 2018, 143 (2), pp.151-167. 10.3917/top.143.0151 . hal-02530091

\section{HAL Id: hal-02530091 \\ https://hal-univ-paris.archives-ouvertes.fr/hal-02530091}

Submitted on 7 Apr 2020

HAL is a multi-disciplinary open access archive for the deposit and dissemination of scientific research documents, whether they are published or not. The documents may come from teaching and research institutions in France or abroad, or from public or private research centers.
L'archive ouverte pluridisciplinaire HAL, est destinée au dépôt et à la diffusion de documents scientifiques de niveau recherche, publiés ou non, émanant des établissements d'enseignement et de recherche français ou étrangers, des laboratoires publics ou privés. 


\title{
Violences conjugales, famille, vulnérabilité
}

\author{
Laurie Laufer \\ Thamy Ayouch
}

D'un point de vue analytique, s'interroger sur les violences conjugales revient à remettre en perspective la question de l'actualité de la pulsion de mort telle que Freud l'a définie dans son texte «Pourquoi la guerre ?» : le paradoxe d'une destructivité où le sujet se sauve en détruisant l'autre. Les violences conjugales procèdent autant d'un drame subjectif que d'une problématique collective, nouant l'intime du sujet à des enjeux sociétaux et politiques, juxtaposant ce qu'il a été convenu de nommer «espace privé» et «espace public», et brouillant ainsi les frontières entre l'individuel et le collectif. Mais y surgit alors un paradoxe pour l'approche analytique : ces violences semblent poser à la clinique et à la théorisation analytiques, de manière amplifiée, la question de la généralisation. Comment, en effet articuler un «savoir» psychanalytique de la violence, en saisissant toutefois au cas par cas chaque situation?

La particularité des violences conjugales est de s'inscrire au sein d'un dispositif, le couple, où préside un choix amoureux et consenti (nous ne parlerons pas de ce que l'on appelle mariage forcé). S'interroger sur ces violences revient à tenter de penser les impasses et les ressources actuelles du «couple» et de la famille, comme dispositif dit «intime», social, et politique. C'est également aborder les butées de l'amour avec ses normes idéales, ses illusions décevantes et ses passions tristes mais aussi ses ressorts, ses passions joyeuses et ses potentialités. Néanmoins, statuer analytiquement des processus subjectifs ou de couple «typiques », des «profils» de situations de violence de couple, des étiologies généralisables, ou une nosographie spécifique («le sujet pervers », «le masochisme féminin »), revient à perpétrer la violence d'une négation de la singularité subjective et à faire fonctionner ici le savoir analytique en discours dogmatique 
prétendant rendre compte de manière unitaire, voire universelle, d'une réalité singulière. La violence conjugale s'inscrit donc dans la spécificité de la tension propre à la rencontre subjective de deux personnes particulières, rencontre qui, malgré sa ressemblance à d'autres, n'y est pas superposable. Les analystes n'ont de cesse de rappeler cette hypersingularité : c'est toutefois en son nom qu'est le plus clair du temps refusée la prise en compte d'un contexte sociétal, jugé trop général, trop extérieur au sujet, trop sociologisant pour une psychanalyse dite ou imaginée «authentique».

La position est ici surprenante: au nom de la singularité du sujet, on refuse de prendre en compte l'inscription des violences conjugales dans le contexte irréductible, bien que général, d'oppression de genre («c'est de la militance, et non pas de la psychanalyse », peut-on souvent entendre). Mais en même temps, faisant fi de cette hypersingularité, bien des analystes s'escriment à comprendre les situations de violences conjugales par concrétions générales («érotisation de la souffrance », « jouissance Autre », « espoir de changer son homme», « identification à la bonne mère », etc.). Et ces concrétions s'exemptent de toute analyse de la façon dont fonctionne en leur sein, une structure d'oppression de genre, un système sexe/genre particulier.

Comment opère donc le genre dans l'approche clinique et théorique des violences conjugales? Y répondre convoque probablement une prise en compte des vulnérabilités qui traversent les violences conjugales, pour penser les conditions de possibilités d'une puissance d'agir, tant individuelle que collective, au sein de laquelle il sera judicieux de différencier l'acte de l'action.

Pour aborder les violences conjugales dans leur dimension à la fois sociale, politique et hypersingulière, il semble alors pertinent de considérer d'abord les effets paradoxaux d'une certaine psychopathologie de la violence conjugale. Considérant que «la psychologie individuelle est une psychologie sociale» (Freud), nous opposerons à ces approches la double inscription des violences conjugales dans des violences de genre: violences sociétales et dispositif de la famille, mais aussi violences propres à une certaine clinique et théorisation analytiques. Il s'agira enfin de tenter de penser la pratique analytique dans ces contextes en mettant en exergue la vulnérabilité opérant au sein de la subjectivation, «la transformation érotico-sociale » (Freud), et la manière dont la clinique analytique la considérant s'avère politique.

\section{I.}

Qu'est-ce qui pousse un sujet à vouloir en détruire un autre? Qu'est ce qui fonde la haine et la destructivité ? Selon Freud, la violence sociale est la plus dévastatrice pour la vie psychique. En 1920, il émet l'hypothèse de la pulsion de mort et de la compulsion de répétition. Si auparavant la répétition avait pour 
FAMILLE, VULNÉRABILITÉ

fonction de réguler, voire de diminuer le traumatisme, il est amené à constater qu'elle ne parvient pas à remplir cette mission, et doit sans cesse être reconduite : elle ne cesse de ne pas cesser. La répétition peut alors apparaître, souvent comme un phénomène d'emprise, la production d'un lien paradoxal par lequel un sujet en détruit un autre pour le conserver. Entre l'amour et la haine, entre Éros et Thanatos, qui peut prévoir l'issue du combat? Telle est l'ultime question que Freud ajoute à son texte, en 1931, dans la deuxième édition de Malaise dans la civilisation.

Si dans Totem et Tabou Freud avance l'idée que l'essence même de la famille est d'être un meurtre, lorsqu'il développe cette hypothèse, il considère davantage la haine et la violence des enfants sur leurs parents (mythe œdipien des vœux incestueux et meurtriers) que celles advenant dans le couple lui-même, et vise plutôt les violences psychiques que physiques. Que peut expliquer la violence physique et psychique faite aux enfants ou/et aux femmes? Quel amour, quelle haine, quelles motions pulsionnelles peuvent justifier un passage à l'acte violent (agression sexuelle, coups entamant l'intégrité physique) sur le corps d'une femme?

Il existe une psychopathologie différentielle des actes violents, établie à partir de la jouissance qu'ils comportent - une jouissance qui emporte le sujet dans le flux de sa destructivité. Cette psychopathologie est toutefois à comprendre au cas par cas dans des contextes variés. Les causalités et multiples étiologies sur les (dé)raisons d'un acte de violence peuvent momentanément avoir des effets d'apaisement sur l'angoisse que suscite l'horreur de la situation. Comprendre, donner un sens aux causes de la violence peut parfois réduire l'angoisse du/de la clinicien/ne, des victimes ou des auteur/es de violences eux/elles-mêmes. Donner un sens à un acte in-sensé l'intègre dans un récit dont la vertu imaginaire fait écran au Réel.

Cette imaginarisation ne manque pas, en outre, de reprendre des représentations de genre non analysées, que bien des auteur/es mettent au fondement de leurs étiologies et nosographies en refusant autant la politisation des questions de genre et de sexualité que celle de la psychanalyse. La procédure consistant à nier toute inscription politique est toutefois, malgré qu'elle en ait, elle-même politique : elle choisit une répartition des jeux de pouvoir décidant de l'ordre et de la conservation contre le changement. Faisant de l'intime ou du privé qu'aborde la psychanalyse un lieu hors polis, elle maintient ininterrogés les rapports de pouvoir qui le structurent. Sous couvert d'interroger la responsabilité du sujet dans ce qui lui arrive et l'étrange satisfaction qu'il tire de situations de violence, nombre de théorisations analytiques se mettent ainsi en passe de définir une «jouissance féminine» au centre des violences conjugales et de leur maintien. Et de rappeler bien des phrases que ceux/celles qui les colportent sans les contextualiser ou les remettre en perspective historique ont transformées en ritournelle dogmatique : 
Chez Lacan :

«Si la femme est le symptôme de l'homme, l'homme n'est pas le symptôme de la femme : il en est son ravage ${ }^{1} »$,

ou encore :

«Il n'y a pas de limites aux concessions que chacune fait pour un homme: de son corps, de son âme, de ses biens ${ }^{2}$ »

ou chez Freud

«La sujétion est incomparablement plus fréquente et plus intense chez la femme que chez l'homme ${ }^{3} »$

ou encore chez H. Deutsch :

«Le goût du malheur est incomparablement plus grand chez les femmes que chez les hommes ${ }^{4} »$.

Si ces affirmations présentent quelque sens à un moment et dans une aire culturelle donnés, on est en droit de se demander comment leur valeur descriptive ponctuelle devient prescriptive d'un mode, outrancièrement généralisé, de subjectivation de «la» femme, en tout temps et tout lieu ... Comment, en outre, le fait de se cantonner à les ânonner ne conduit pas non seulement à les faire fonctionner performativement, mais également à ne chercher en rien à modifier cette configuration de genre (jugée immuable, universelle, a-historique).

On s'empresse alors de désigner, comme le fait 1'un ${ }^{5}$, des mécanismes psychiques universellement féminins :

«a satisfaction d'un besoin de punition;

la demande d'amour paternel sans limites ;

l'identification à la bonne mère et à la mère maltraitée ;

la fétichisation de la puissance masculine;

ou le penchant de beaucoup de femmes à répondre positivement au

désir de l'Autré6.»

1. J. Lacan, Sémininaire sur le Sinthome, séance du 17 février 1976, cité par P. de Neuter, «Pourquoi un homme est-il si souvent un ravage pour sa femme», in M.-J. Grihom, M. Grollier, Femmes victimes de violences conjugales. Une approche clinique, Rennes, Presses Universitaires de Rennes, 2012, p 33.

2. J. Lacan, Télévision, Le Seuil, 1973, p 63-64, cité par P. de Neuter, «Pourquoi un homme est-il si souvent un ravage pour sa femme », in M.-J. Grihom, M. Grollier, Femmes victimes de violences conjugales. Une approche clinique, op. cit., p 33, ou par M. Grollier «Folie dans le couple et la famille : un amour qui ne fait pas condescendre la jouissance», ibid., p 80.

3. S. Freud, «Le tabou de la virginité», in La Vie sexuelle, PUF, 1969, p 67, cité par P. de Neuter, op. cit., p 33.

4. H. Deutsch, La psychologie des femmes, PUF, 1949, p 235, cit par P. de Neuter, op. cit., ibid.

5. P. de Neuter, op. cit., p 35 .

6. Ibid. 
LAURIE LAUFER, THAMY AYOUCH - VIOLENCES CONJUGALES, FAMILLE, VULNÉRABILITÉ

Et de rappeler que «la petite fille ne subit pas la menace de castration, ce qui fait qu'elle ne renonce jamais complètement à sa demande d'amour au père. Il s'ensuit que tout Autre auquel elle adressera sa demande d'amour dans l'avenir pourra la soumettre à des exigences éventuellement limitées ${ }^{7}$ », ou que «de nombreuses femmes recherchent la compagnie d'homme puissants, des hommes «qui en ont», façon pour elles d'être possesseures du phallus par l'intermédiaire de leur homme ${ }^{8} » \ldots$

Un autre auteur reprend à son compte l'analyse lévi-straussienne de l'échange des femmes comme origine de la culture, et sa relecture lacanienne, qui situe les femmes à la fois comme sujet de l'inconscient et objet de l'échange9. Bien mal avisé/e qui ici dirait, à la manière de Gayle Rubin, que l'échange des femmes ne constitue ni une définition de la culture ni une réalité ontologique immuable, mais caractérise un système de parenté historiquement situé, qui dévoile certains aspects des rapports sociaux de sexe et de genre ${ }^{\mathbf{1 0}}$. De ce statut de femme objet de l'échange, on conclut au destin mortifère inéluctable d'objet pour le fantasme d'un homme ${ }^{11}$, qui confine «la» femme à la position de jouissance Autre. Ces femmes, alors, « ne peuvent trouver le point d'arrêt sur la pente du pire ${ }^{12} \gg$ :

«Dans cette position où elle a une jouissance incernable, sans limite de par le fait qu'elle n'est pas tout entière soumise à la castration, une femme peut alors se prêter sans limite à un homme et attendre de lui qu'il lui donne ses limites ${ }^{13}$.

C'est donc par manque de phallicisation de leur jouissance qu'elles pêchent, et l'on entend le leur signaler à coup de raclées...

D'autres lient la souffrance d'une femme dans son couple à son incapacité de «remplir les fonctions de femme idéale», femme non-manquante, toute1 1 ces préconçus théoriques sont alors plaqués sur les situations cliniques les plus diverses. Ces acrobaties improbables entre un psittacisme théorique et des situations cliniques déformées pour la cause ne pourraient concerner, après tout, que leurs auteur/es. Mais outre l'impéritie théorico-clinique ici affichée, menace le danger d'une surdité à la clinique, de sa totale dépolitisation, et d'un renforcement des normes de genre. L'analyste apparaît ici comme celui ou celle qui

7. Ibid., p 36

8. Ibid., p 37.

9. M. Grollier «Folie dans le couple et la famille: un amour qui ne fait pas condescendre la jouissance », ibid., p 76.

10. G. Rubin, «Économie politique» du sexe, et systèmes de sexe/genre, in Surveiller et jouir. Anthropologie politique du sexe, EPEL, 2010, p 45.

11. M. Grollier, op. cit., p 76.

12. Ibid., p 78.

13. Ibid., p 81. 
renaturalise la différence des sexes, et cantonne les femmes à une irréductible position de victime et les hommes à celle de l'agresseur.

Face à ces ontologisations de traits de genre, et dans une perspective s'attachant à considérer les violences conjugales dans leur dimension relationnelle, d'autres auteurs s'accordent à interpréter le déchaînement de la violence comme un acte de défense interne contre l'angoisse de perdre l'autre et le pouvoir qu'on a sur lui/elle. Cette violence devient une nécessité paradoxale pour conserver un équilibre social et un équilibre interne: détruire l'autre pour le/a conserver. Freud, dans les Trois essais parle de «pulsion d'emprise»(Bemächtigungstrieb) ${ }^{\mathbf{1 5}}$ : prendre pour échapper à la prise, sortir de la prise en prenant. Le sujet s'empare d'un/e autre pour lui faire subir ce qu'il/elle a subi lui-même. En mettant l'autre à la place qui a été la sienne, le sujet veut assumer une position active par rapport à ce qu'il a subi passivement: effets incessants de renversements de positions. Dans cette boucle pulsionnelle où s'articulent intrication et désintrication, l'enjeu est la répétition même, répétition qui se joue des acteurs. La violence entre dans cette boucle, et les deux protagonistes se retrouvent, chacun/e à son tour, dans la position de bourreau et de victime. Il n'y a pas de dominant/e et de dominé/e : les deux sont également soumis/es, non au/à la partenaire, mais au mouvement circulaire dans lequel ils/elles sont pris/es. On peut mettre en perspective une série de paradoxes pour tenter de comprendre cette violence: détruire l'autre pour le/a conserver, idéaliser l'objet tout en déchaînant sa cruauté contre lui lorsque cette idéalisation est menacée ou lorsqu'elle s'effondre, aimer et haïr dans un mouvement simultané, se soumettre à une pulsion de domination. Ces hypothèses donnent un sens à des scènes qui sont hors scènes ou obscènes. Cependant, comme l'écrit Jacques Hassoun :

«La psychanalyse nous l'enseigne, les données de sens et d'évidence relèvent de l'imaginaire. Ce sont des scènes, des scénarios qui peuvent permettre une certaine articulation du Symbolique et du Réel. Mais lorsqu'elles sont fixées, figées, enkystées, fétichisées, elles constituent au contraire un obstacle à la symbolisation ${ }^{\mathbf{1 6}}$.»

Une autre voie d'approche psychopathologique relève du paradigme de la mélancolie : une indignité du sujet qui considère qu'il ne vaut pas mieux que la situation qu'il est en train de vivre. La mélancolie en tant que souffrance sans angoisse est la disparition du sujet, son effacement, une désubjectivation.

Elle se manifeste par une impossibilité de métamorphose. Chacun/e dans la violence est une proie ; l'un/e la proie de l'autre, l'autre la proie de son acte. La

14. Magali Curato, Thierry Bisson, «Violences conjugales et couples de victimes-agresseurs. Être victime dans son couple, victime de qui, de quoi ?», in op. cit., p 126.

15. S. Freud, Trois essais sur la théorie sexuelle, Paris, Folio, 1985, 114.

16. J. Hassoun, Actualités d'un malaise, Paris, Erès, 1999, p. 111. 
LAURIE LAUFER, THAMY AYOUCH - VIOLENCES CONJUGALES, FAMILLE, VULNÉRABILITÉ

passivité de la mélancolie est souvent déclenchée par une situation qui dépasse le sujet et le confronte à l'impossibilité à réagir face à l'événement. C'est en ces termes que Elias Canetti en rend compte :

«La mélancolie commence quand les métamorphoses de fuite sont achevées et qu'on en éprouve l'inutilité. Dans la mélancolie, on est la proie rattrapée et déjà saisie. On ne peut plus s'échapper. On ne se transforme plus. Tout ce qui a été essayé a été vain. On s'est résigné à son sort, on se voit proie ${ }^{17}$. »

À ce propos, la notion freudienne de mélancolie pourrait être rapprochée du concept de «mélancolie de genre», développé par Judith Butler dans Troubles dans le genre, mais surtout dans La Vie psychique du pouvoir, et issu lui-même d'une théorisation analytique. Si, comme nous avons tenté de le montrer, la violence n'est pas genrée, à un niveau inconscient, et, partant, dans les métapsychologies qui tentent d'en rendre compte, elle ne s'inscrit toutefois pas moins dans un contexte sociétal, où une configuration particulière des relations de genre ne manque pas d'influencer les positionnements subjectifs. Toute la différence consiste ici à ne pas considérer certains processus psychiques comme constituants anhistoriques d' «identités » de genre. La mélancolie de genre désigne l'opération par laquelle une partie du pouvoir érogène du corps est définitivement perdue, devient, de ce fait, une impossibilité performative et fabrique ainsi un corps genré. Un processus naturalisé d'hétérosexualisation des corps produit, selon J. Butler, les limites du réel : les faits psychiques y servent alors de cause et les désirs d'effets de cette nature physique ${ }^{\mathbf{1 8}}$. Cette perte d'une partie du pouvoir érogène du corps est déniée, et la surface sexuée du corps émerge comme le signe d'une identité (de genre) et d'un désir naturalisés. Cela revient alors à considérer le genre, contrairement à la logique de l'identité, non point comme affirmation mais comme processus social et psychique de privation (de postures psychiques de sexuation et de sexualité) : la sexuation genrée apparaît comme forclusion d'une multiplicité psychique et forclusion d'une hybridité des genres et des sexualités.

Nous soutiendrions donc que certaines situations de violence conjugale viennent de postures subjectives tentant de se décoller de ces assignations de genre, de défaire la captation imaginaire qu'elles introduisent au sein d'un couple. S'y inscrire durablement, ne pas parvenir à s'en extraire, semble toutefois relever d'un enkystement de la mélancolie de genre, de son renforcement par la mélancolie rendant impossible la métamorphose ou l'action.

On a pu dire des victimes qu'elles étaient masochistes $\bar{\equiv}$ ives, les enfermer

17. E. Canetti, Masse et Puissance, Paris, Gallimard, 1966, p. 368.

18. J. Butler, Troubles dans le genre. Le féminisme et la subversion de l'identité, Paris, La découverte, 2005 p 167. 
en somme dans un discours psychopathologique qui a aussi comme effet de les condamner, de naturaliser leurs comportements. Comment sortir de la passivation, transformer la mélancolie, cette souffrance sans angoisse ? La violence relève du Réel, d'un impossible à nommer : s'il s'agit de tenter de comprendre les ressorts de cette impasse, il s'agit aussi de ne pas tomber dans le piège d'une production de sens incessante. $\mathrm{Si}$, en outre, la violence conjugale peut relever d'une psychopathologie, elle s'inscrit aussi dans un dispositif social, et des assignations de genre.

II.

La psychologie individuelle est une psychologie sociale comme le souligne Freud :

«Dans la vie psychique de l'individu pris isolément, l'Autre intervient très régulièrement en tant que modèle, soutien et adversaire, et de ce fait la psychologie individuelle est aussi d'emblée et simultanément une psychologie sociale ${ }^{19}$ 》)

Contre toute essentialisation de « traits féminins », et eu égard à l'inscription des processus de subjectivation des femmes dans des conditions sociétales, Freud écrit également:

«Il nous faut prendre garde de ne pas sous-estimer l'influence des organisations sociales qui acculent également la femme à des situations passives. Tout cela est encore loin d'être tiré au clair...La répression de son agressivité, constitutionnellement prescrite et socialement imposée à la femme, favorise le développement de fortes motions masochistes qui parviennent à lier érotiquement les tendances destructrices tournées vers le dedans ${ }^{20} \ldots »$.

Il convient donc de souligner l'irréductible dimension de violence sociétale de genre dans laquelle s'inscrivent les violences conjugales. Mise en exergue par les discours féministes des années 70, elle est révélée au grand jour en 2000, en France, par l'enquête ENVEFF. Un des apports de celle-ci a été de montrer que dans le cadre conjugal en particulier, la perception de la violence n'est pas toujours immédiate. Outre à la peur, à la honte, voire à une forme de culpabilité, l'ampleur du silence tient à ceci que le système sexe-genre dans lequel s'inscrivent ces violences n'est pas en soi contesté-il est considéré comme naturel, inéluctable et non transformable.

19. S. Freud, Essais de psychanalyse, Paris, Payot, 1981, p. 124.

20. S. Freud, «La féminité », Nouvelles conférences d'introduction à la psychanalyse, Paris, Gallimard, 2002, p. 177. 
Comme le soulignent Natacha Chetcuti et Maryse Jaspard dans Violences envers les femmes: Trois pas en avant deux pas en arrière, les violences contre les femmes s'inscrivent donc, en premier lieu, dans un système sexe/genre de hiérarchisation des rapports sociaux de sexe, et notamment de domination masculine. L'approche féministe de ces violences révèle leur irréductible dimension politique : elles résultent d'un mécanisme de contrôle social maintenant la subordination des femmes par les hommes. Il est donc fondamental de ne pas escamoter cette dimension socio-politique du phénomène en considérant ces violences exclusivement comme un problème de justice, de sécurité intérieure ou encore de santé publique, relatif à une psychopathologie qui circonscrirait la question dans la seule psychologisation de la victime ou de l'auteur. La violence conjugale n'est pas une pratique isolée ou aberrante : elle prend sens dans un système de genre particulier, sur lequel elle s'appuie et qu'elle renforce en retour, système construit, et donc susceptible d'être modifié. Si l'approche analytique peut s'avérer ici utile, c'est en tant qu'elle permet de penser et de déconstruire la manière dont des modes de subjectivation de femmes ou d'hommes s'inscrivent dans un assujettissement particulier à des normes de genre.

De ces « organisations sociales qui acculent les femmes à une situation de passivitée ${ }^{11}$, la famille fait partie. La famille est un dispositif construit socialement qui répond aux normes d'une époque: devenu noyau sécurisant et protecteur à partir de la naissance du biopolitique, elle relève d'un discours normatif.

Dans la présentation de Le soi, le couple et la famille le sociologue François de Singly écrit :

«Oui, la famille a changé. Non seulement son cadre institutionnel a craqué, mais sa fonction centrale s'est également modifiée. Son rôle premier a longtemps été la transmission du patrimoine économique et moral, d'une génération à l'autre. Aujourd'hui la famille tend à privilégier la construction de l'identité personnelle, aussi bien dans les relations conjugales que dans celles entre parents et enfants.»

C'est en somme ce que Michel Foucault avait déjà analysé dans Le Pouvoir psychiatrique puis dans la Volonté de savoir. Dans le cours au Collège de France de 1973-74, la famille apparaît comme pièce centrale à la fois dans le pouvoir disciplinaire, et dans la théorisation psychanalytique. Elle cristallise la rémanence du pouvoir souverain dans la société disciplinaire. Précisément parce qu'elle n'obéit pas à un schéma disciplinaire, mais à un dispositif de souveraineté, elle constitue, pour Foucault, la charnière de tous les systèmes discipli-

21. Ibid. 
naires, l'instance de contrainte qui fixe les individus sur les appareils disciplinaires ${ }^{22}$. Lorsque la famille menace de se délabrer et de ne plus jouer sa fonction apparaissent une série de dispositifs disciplinaires visant à pallier cette défaillance : maisons pour les enfants trouvés, orphelinats, maisons pour jeunes délinquants $^{23}$. La familiarisation du milieu thérapeutique de la maison de santé se double d'une disciplinarisation de la famille, qui devient une instance de désignations des individus anomaux 22

C'est alors la fonction-psy, incarnée de manière égale, selon Foucault, par les psychologues, psychothérapeutes, psychopathologues, criminologues et psychanalystes, qui opère le branchement des dispositifs disciplinaires sur la souveraineté familiale ${ }^{25}$. Elle articule le caractère indisciplinable de l'individu à une défaillance familiale, et assure ainsi les schémas d'individuation, de normalisation et d'assujettissement des individus à l'intérieur des systèmes disciplinaires. C'est pourquoi, conclut Foucault, « il n'est pas étonnant que le discours de la famille, le plus « discours de famille» de tous les discours psychologiques, c'est-à-dire la psychanalyse, puisse, à partir du milieu du $\mathrm{XX}^{\mathrm{e}}$ siècle, fonctionner comme étant le discours de vérité à partir duquel on peut faire l'analyse de toutes les institutions disciplinaires ${ }^{\mathbf{2 6}} \gg$.

Foucault reconduit cette analyse dans La Volonté de savoir, où il appréhende la famille comme l'échangeur du dispositif de sexualité et du dispositif d'alliance ${ }^{27}$. Le dispositif de sexualité, qui permettait de gérer un patrimoine, une filiation, et transmettre une citoyenneté, s'était développé auparavant dans les parages de la famille; il se recentre sur elle avec la psychanalyse :

«Ce qui s'est passé depuis le XVIII ${ }^{\mathrm{e}}$ siècle peut se déchiffrer ainsi : le dispositif de sexualité, qui s'était développé d'abord dans les marges des institutions familiales (dans la direction de conscience, dans la pédagogie), va se recentrer peu à peu sur la famille : ce qu'il pouvait comporter d'étranger, d'irréductible, de périlleux peut-être pour le dispositif d'alliance - . . est repris en compte par la famille - une famille réorganisée, resserrée sans doute, intensifiée à coup sûr par rapport aux anciennes fonctions qu'elle exerçait dans le dispositif d'alliance. Les parents, les conjoints deviennent dans la famille les principaux agents d'un dispositif de sexualité qui à l'extérieur s'appuie sur les médecins, les pédagogues, plus tard les psychiatres, et qui à l'intérieur vient doubler

22. M. Foucault, Le Pouvoir psychiatrique, op. cit., p 81.

23. Ibid., p 86.

24. Ibid., p 115 .

25. Ibid., p 86.

26. Ibid., p 88.

27. M. Foucault, Histoire de la sexualité. Tome I. La Volonté de savoir, Paris, Gallimard, 1976, p 148-149. 
LAURIE LAUFER, THAMY AYOUCH - VIOLENCES CONJUGALES, FAMILLE, VULNÉRABILITÉ

et bientôt «psychologiser» ou «psychiatriser» les rapports d'alliance. Apparaissent alors ces personnages nouveaux: la femme nerveuse, l'épouse frigide, la mère indifférente ou assiégée d'obsessions meurtrières, le mari impuissant, sadique, pervers, la fille hystérique ou neurasthénique, l'enfant précoce et déjà épuisé, le jeune homosexuel qui refuse le mariage ou néglige sa femme ${ }^{28} »$.

Découvrant au cœur de la sexualité, comme principe de sa formation, la loi de l'alliance (la parenté, l'interdit de l'inceste), la psychanalyse épingle le dispositif de sexualité sur celui de l'alliance ${ }^{29}$. On retrouve ici incontestablement les positions actuelles de nombre de psychanalystes défenseur/es de «l'ordre symbolique » de la famille. Mais outre dans leurs propos millénaristes, le familialisme de la psychanalyse apparaît clairement dans l'imaginarisation de son appareillage théorique: la démétaphorisation ou littéralisation des Edipe, scène primitive, Nom-du-Père et différence des sexes.

Si donc la famille est le « lieu obligatoire d'affects, de sentiments, d'amour», si «la sexualité a pour point privilégié d'éclosion la famille ${ }^{30}$ », cette obligation devient une contrainte et un devoir persécuteurs: la chute de l'astre idéalisé qu'est la famille est alors désastre. Car qu'est-ce qu'un lieu qui ne donne place à personne, où se joue une absence de reconnaissance et une lutte à mort des places et des désirs ? Le lieu obligatoire où il s'agit de tout faire : éduquer, cultiver, produire, faire l'amour.

Comme l'écrit Luc Boltansky, «plus le persécuteur est proche, plus les blessures et les offenses qu'il inflige, et qui, en l'absence de prise en charge collective, ne peuvent être formulées dans un discours normal, sont endogénéisées, et plus elles ont pour effet d'attaquer l'intégrité de la victime et, en quelque sorte, de l'arracher à elle-même ${ }^{31}$ ».

Dans cette proximité, il y a un dispositif de reproduction des normes de genre et des stéréotypes des rôles sexués. Le déclenchement des disputes, conflits qui peuvent dégénérer en violence, résulte souvent de ces reproductions de normes (fantasmes de virilité et masculinité, tâches domestiques, éducation des enfants, indépendance et dépendance économique, valorisation et dévalorisation du rôle social par le travail, etc.). Ces dominations de genre peuvent être complexifiées par des logiques intersectionnelles (statuts sociaux, origine culturelle).

28. Ibid

29. Ibid

30. M. Foucault, op. cit., p 143.

31. Luc Boltanski, L'amour et la justice comme compétences : trois essais de sociologie de l'action, 1990, p. 293. 
La violence qui relève du Réel, impossible, innommable, insensé (pure culture de la pulsion de mort), lorsqu'elle devient violence conjugale est aussi à comprendre du côté de normes sociales et ces normes relèvent de l'imaginaire:

«La psychologie est véhicule d'idéaux, écrivait Lacan en 1966. La psyché n'y représente plus que le parrainage qui la fait qualifier d'académique. L'idéal est serf de la société. Un certain progrès de la nôtre illustre la chose, quand la psychologie ne fournit pas seulement aux voies, mais défère aux vœux de l'étude de marché32.»

La famille et le couple comme dispositifs normatifs sont véhicules d'idéaux et les sujets deviennent les esclaves, les serfs de ces normes sociales.

Comment alors créer les conditions de la transformation de cette violence, autant sociale que subjective et relationnelle, comment le dénouement est-il possible?

III.

La violence conjugale repose en partie sur les conditions sociales de réception des discours sur la violence. Celles-ci produisent les conditions d'émergence de récits et permettent de rendre publiques ces violences, de politiser le phénomène et peut-être alors de déplacer les lignes de partage entre le tolérable et l'intolérable.

Il y a donc d'abord ce qui relève de la justice qui intervient comme tiers, comme rappel à la loi sociale. Si le viol est reconnu comme crime en 1989, et le harcèlement sexuel au travail en 1992, la prise en compte au plan juridique des violences conjugales est bien plus tardive. La prévention et répression des violences au sein du couple ou contre les mineur/es est institutionnalisée par la loi du 4 avril 2006, puis par celle du 5 mars 2007 prévoyant un suivi sociojudiciaire contre les auteurs de violences conjugales. La loi du 9 juillet 2010 relative aux violences faites spécifiquement aux femmes crée une ordonnance de protection pour les victimes de violences conjugales qui combine des mesures civiles et pénales, et reconnaît le délit de harcèlement moral au sein du couple, puis la loi du 4 août 2014 pour l'égalité réelle entre les femmes et les hommes renforce le dispositif des ordonnances de protection.

En outre, depuis un décret de 2016, il existe un stage de responsabilisation dédié aux auteurs de violences conjugales. En mesure alternative aux

32. Lacan Jacques, (1964), «Position de l'inconscient», in Écrits, Paris, Seuil, 1966, p. 832. C'est moi qui souligne. 
LAURIE LAUFER, THAMY AYOUCH - VIOLENCES CONJUGALES, FAMILLE, VULNÉRABILITÉ

poursuites, dans le cadre de la composition pénale ou suite à une décision de jugement, c'est un stage d'une journée co-animé par un/e psychologue et un/e intervenant socio-judiciaire. Il est décrit en ces termes sur le site du Sénat:

«L'article 50 de la loi du 4 août 2014 a étendu les stages de sensibilisation, déjà prévus par exemple pour les auteurs de violence parentale, les auteurs d'infractions au code de la route et les toxicomanes, aux auteurs de violences conjugales. Elle a donné un fondement législatif à des stages qui existaient déjà et auxquels elle a conféré la dénomination officielle de «stages de responsabilisation pour la prévention et la lutte contre les violences au sein du couple».»

Ces stages peuvent être une alternative aux poursuites. Ils sont décidés par le procureur de la République et effectués aux frais de l'auteur des faits.

Les objectifs sont de permettre aux stagiaires de réfléchir sur les principes fondamentaux de la vie en société et de comprendre la portée de leurs gestes $^{33}$.

Si les pouvoirs publics peuvent intervenir sur les actes, un travail de la parole, auprès de psychologues et psychanalystes, entend permettre des effets de transformation ou de refoulement. Les dispositifs de judiciarisation opèrent du côté de la représsion des actes; le travail du refoulement relèverait des conditions d'une tâche «érotico-sociale» et thérapeutique. Il reste en effet important de répondre aux violences conjugales non seulement sur le plan du pouvoir juridique, par des mesures judiciaires, mais également sur celui du pouvoir disciplinaire et, pour ce qui concerne les analystes, des effets de subjectivation qui en résultent.

Nous concevons, de manière foucaldienne, les processus de subjectivation comme assujettissement à des normes, et, en l'occurrence, comme incorporation spécifique de normes de genre. Déconstruire la technologie disciplinaire de subjectivation que favorise le système sexe-genre, c'est commencer par reconnaître la plus grande exposition à la vulnérabilité qu'il instaure. Dans un sens butlerien, la vulnérabilité des corps, leur interdépendance, constitue un aspect de la modalité sociale à travers lesquelles ils subsistent ${ }^{34}$. Elle peut-être toutefois accentuée et manipulée dans le processus de production et de naturalisation de formes d'inégalité sociale. La vulnérabilité ne connaît donc pas de distribution sociale égale : certain/es, pour leur situation de genre, de classe, de race, sont

33. LOI n ${ }^{\circ}$ 2014-873 du 4 août 2014 pour l'égalité réelle entre les femmes et les hommes (1) NOR : FVJX1313602L

ELI : https ://www.legifrance.gouv.fr/eli/loi/2014/8/4/FVJX1313602L/jo/texte

Alias : https ://www.legifrance.gouv.fr/eli/loi/2014/8/4/2014-873/jo/texte

Titre III : Dispositions relatives à la protection des personnes victimes de violences et à la lutte contre les atteintes à la dignité et à l'image à raison du sexe dans le domaine de la communication.

34. J. Butler, Qu'est-ce qu'une vie bonne?, Paris, Payot, 2014, p 91. 
exposé/es à une plus grande vulnérabilité que d'autres. C'est ici le cas des femmes dans un système de sexe/genre patriarcal, encore plus vulnérabilisées si elles sont de couleur, ou lesbiennes, ou trans. Il ne s'agit pas ici de chercher à supprimer la vulnérabilité, propre à l'interdépendance des corps, mais d'écouter les conditions dans lesquelles elle est accentuée, d'en penser la viabilité, et d'en pointer la contingence. C'est alors que peut surgir une déconstruction de la nécessité, construite des processus de subjectivation-assujettissement.

L'écoute de la singularité subjective, pourrait-on alors demander, n'est-elle pas compromise par une analyse qu'on dirait sociale, sociologique et qui ne prendrait pas en compte la spécificité psychique de telle ou telle femme ? Nous croyons que cette spécificité s'inscrit néanmoins dans des conditions sociétales de subjectivation-assujettissement. La visée de l'analyse apparaît alors comme ce qui précisément pointe la contingence de ces processus psychiques et identitaires, conscients mais surtout inconscients. Elle vise à favoriser une plasticité psychique, qui, levant la répétition, fait que ce qui ne pouvait pas advenir autrement prenne d'autres voies.

En outre, la plus grande vulnérabilité ici mise en exergue permet de mieux rendre compte de la dimension dynamique, relationnelle des rapports de violence conjugale. L'exposition à un degré plus élevé de vulnérabilité propre à la violence sociétale de genre permet de substituer au statut essentialisé de victime sociale ou à un profil de jouissance, la notion de «processus de victimisation ». Cette notion met en exergue l'impact, processuel, de la violence vécue, et des défenses mobilisées pour y faire face. Ce qui était auparavant présenté comme processus intrapsychique propre à un sujet, inscrit dans son identité de sexe (sa jouissance ou son masochisme) et érigé comme cause de la situation de violence, devient alors le résultat d'une interaction où l'une des deux est plus vulnérabilisée, et apparaît alors comme conséquence de cette violence.

Si la violence conjugale est un processus relationnel, il convient, en outre, d'en accompagner les auteur/es dans une réflexion sur la portée de leur geste. Cela soulève la question de savoir ce qu'est un passage à l'acte. Si l'acte relève de l'instant, acte et action ne partagent alors pas la même temporalité. L'acte est l'inassimilable, ce qui résiste à la symbolisation, et permet, par décharge immédiate, la satisfaction du principe de plaisir. L'action, elle, s'inscrit dans le temps, elle relève d'un processus, d'un déploiement: penser à ses effets peut permettre au sujet de laisser émerger en lui des affects, notamment de l'angoisse. $\mathrm{Si}$, comme nous le soulignions, la mélancolie est une souffrance sans angoisse, on s'aperçoit que lorsque la violence conjugale s'étend au sein de la famille, sur les enfants, l'angoisse ressurgit et peut permettre une réaction.

Qu'est alors l'émergence d'une responsabilisation, de la responsabilité de son acte, visée par les stages auparavant mentionnés? Si le sujet est en mesure 
LAURIE LAUFER, THAMY AYOUCH - VIOLENCES CONJUGALES, FAMILLE, VULNÉRABILITÉ

de s'approprier son acte, de l'inscrire dans son histoire, de produire une autre logique de l'amour et de la haine, sans doute pourrait émerger un déplacement subjectif. La question n'est pas seulement de produire une répression de l'acte, mais de créer les conditions d'une transformation subjective qui permette au sujet de passer à autre chose, de faire cesser la répétition, de comprendre qu'il est captif de son acte et non de l'autre. Il y a en somme tout un travail sur l'amour à faire : comment nouer les nécessités de la détermination amoureuse à la contingence de la rencontre amoureuse ? Il s'agit ici de l'amour non pas seulement comme captif imaginaire des événements psychologiques du sujet, mais comme lien à l'autre, dans une altérité toujours irréductible aux fantasmes propres.

Il s'agit de penser l'amour comme plaisir et non pas seulement comme la manifestation d'un pouvoir, ou des effets imaginaires du tragique, de s'apercevoir que la sexualité relève aussi du comique, et non plus du tragique, comme le soulignait Lacan ${ }^{35}$. Il s'agit donc de travailler à ce que Freud avait nommé les «transformations érotico-sociales ${ }^{36} »$.

\section{CONCLUSION}

Il semble alors légitime de se demander si une clinique de la violence conjugale n'est pas, au même titre que toute clinique de la vulnérabilité, toujours politique. Peut-on alors faire de la psychanalyse sans politique ? Comme l'écrivait Lacan, « l'inconscient, c'est la politique ${ }^{37}$ » : ce qu'il tente d'en articuler par la logique correspond à « ce qui lie les hommes entre eux, ce qui les oppose ${ }^{38}$ », ensemble de normes historiquement inscrites définissant la subjectivation comme assujettissement et résistance à cet assujettissement. Se pose alors la question de savoir si une écoute analytique n'a pas pour visée de favoriser cette résistance à l'assujettissement, constitutif de la subjectivité. Mais n'est-ce pas là viser quelque chose pour l'analysant/e, à sa place ? Pour y répondre, peut-être conviendrait-il de confronter cette résistance à l'assujettissement (résistance qui le reconfigure, mais ne le supprime jamais) à la résistance en analyse. Il n'y a de résistance que de l'analyste, souligne Lacan. La résistance désigne un point idéal abstrait, créé et ainsi nommé par l'analyste, et qu'il est absurde de vouloir « liquider» en la prêtant tout entière à l'analystant/e :

35. «Ce qui dans le sexuel importe, c'est le comique» Ornicar? Bulletin périodique du champ freudien, 1979,19 , p. 5-9.

36. S. Freud, «Actuelles sur la guerre et la mort» Euvres Complètes vol.XIII, trad. fr., Paris : PUF 1994, p.129-157, p.137.

37. J. Lacan, Le Séminaire. Livre XIV. La logique du fantasme. 1966-67, Séance de 10 mai 1967, sténotypie.

38. Ibid. 
«Il n'y a qu'une seule résistance, c'est la résistance de l'analyste. L'analyste résiste quand il ne comprend pas à quoi il a affaire. Il ne comprend pas à quoi il a affaire quand il croit qu'interpréter, c'est montrer au sujet que ce qu'il désire, c'est tel objet sexuel. Il se trompe. Ce qu'il s'imagine ici être objectif n'est qu'une pure et simple abstraction. C'est lui qui est en état d'inertie et de résistance ${ }^{39}$.»

La résistance est ainsi à mettre au compte de l'analyste, lorsqu'il/elle croit fixer un objectif d'avancée, par son interprétation, et fige ainsi le désir de l'analysant/e dans un objet défini. L'analyste résiste pour autant qu'il/elle s'identifie à un idéal de l'analyse, pure, et refuse que les concepts et textes analytiques ne soient questionnés. La fétichisation des catégories historiquement définies utilisées pour écouter (la jouissance Autre, le masochisme féminin, etc.) traduit donc une résistance de l'analyste, une visée particulière.

D'autre part, l'analyste résiste lorsqu'il/elle fait l'économie d'une interrogation sur l'adresse de son acte: travaille-t-il/elle pour l'ordre social, l'institution, l'ordre symbolique, ou pour son analysant/e ? La prétention de neutralité, d'a-politisime, est elle aussi une résistance de l'analyste.

Favoriser donc chez l'analysant/e une résistance à l'assujettissement n'est pas confondre ici la cure avec un programme de militance, mais accompagner l'analysant/e dans la découverte de la contingence des modalités de sa subjectivation-assujettissement, et de la possibilité de sa reconfiguration, et de sa ressignification. C'est l'accompagner dans la déconstruction des normes, sociales et collectives et de la manière dont elles le/a subjectivent, dans l'appréhension de leur contingence.

«Il est certain que se coltiner la misère, comme vous dites, écrivait Lacan, c'est entrer dans le discours qui la conditionne, ne serait-ce qu'au titre d'y protester ${ }^{40}$. $\gg$ Continuons donc de protester contre ces violences en déconstruisant ce qu'il en est des normes familiales, des normes de l'amour et de celles des genres.

Laurie LAUFER laurie.laufer@wanadoo.fr

Thamy AYOUCH

thamy.ayouch@gmail.com

39. Ibid.

40. J. Lacan, Télévision, Paris, Éd. du Seuil, 1973, p. 25. 
FAMILLE, VULNÉRABILITÉ

\section{Laurie Laufer, Thamy Ayouch - Violences conjugales, famille, vulnérabilité}

Résumé: Les violences conjugales procèdent autant d'un drame subjectif que d'une problématique collective, nouant l'intime du sujet à des enjeux sociétaux et politiques, et brouillant ainsi les frontières entre l'individuel et le collectif. Comment alors articuler un «savoir» psychanalytique de la violence, en saisissant toutefois au cas par cas chaque situation? Comment, en outre opère le genre dans l'approche clinique et théorique des violences conjugales? Pour aborder les violences conjugales dans leur dimension à la fois sociale, politique et hypersingulière, les auteur/es commencent par considérer les effets paradoxaux d'une certaine psychopathologie de la violence conjugale. Considérant que «la psychologie individuelle est une psychologie sociale» (Freud), ils/elles opposent à ces approches la double inscription des violences conjugales dans des violences de genre : violences sociétales et dispositif de la famille, mais aussi violences propres à une certaine clinique et théorisation analytiques. L'article s'essaie alors à penser la pratique analytique dans ces contextes en mettant en exergue la vulnérabilité opérant au sein de la subjectivation, conçue comme assujettisement à des normes de genre, et «la transformation éroticosociale» (Freud) visée par une clinique qui, en considérant les modalités de puissance d'agir, s'avère politique.

Mots-clés : Violences conjugales - Violences de genre - Famille - Psychanalyse Vulnérabilité - Puissance d'agir.

Laurie Laufer, Thamy Ayouch - Domestic Abuse, Family, Vulnerability - a Psychoanalytical Approach.

Abstract : Domestic abuse has both a subjective and collective dimension, intertwining the individual with social and political issues, and blurring the frontiers between the individual and the collective. How can one develop a psychoanalytical conception of domestic abuse, while still proceeding on a case-by-case basis? How, moreover, does gender interfere in clinical and theoretical approaches to domestic abuse? In order to tackle the social, political and hypersingular aspects of domestic abuse, the authors first consider the paradoxical effects of a certain psychopathology of domestic abuse. On the grounds that "individual psychology is a social psychology" (Freud), they confront this psychopathology with the gender violence that defines domestic abuse and consists both in social and family violence and the clinical and theoretical violence fostered by certain psychoanalytical approaches. Last but not least, they try to define a psychoanalytical approach that might take into account the vulnerability that characterizes subjectivation, conceived as a subjection to gender norms. A clinical approach aiming for an "erotico-social transformation" (Freud) and new modes of agency proves in this light to be highly political.

Key-words : Domestic abuse - Gender violence - Family - Psychoanalysis Vulnerability - Agency 